\title{
Algorithms and Challenges on the GeoWeb
}

\author{
Radu Jurca \\ Google Maps Zurich \\ radu.jurca@gmail.com
}

\begin{abstract}
A substantial number of queries addressed nowadays to online search engines have a geographical dimension. People look up addresses on a map, but are also interested in events happening nearby, or inquire information about products, shops or attractions in a particular area. It is no longer enough to index and display geographical information; one should instead geographically organize the world's information. This is the mission of Google's GeoWeb, and several teams inside Google focus on solving this problem. This talk gives an overview of the main challenges characterizing this endeavor, and offers a glimpse into some of the solutions we built.
\end{abstract}

